\title{
Delayed cervical somatosensory potentials in cervical spondylosis
}

\author{
E. E L NEGA M Y A N D E. M. SEDGW I C K \\ From the Department of Clinical Neurophysiology, Wessex Neurological Centre, \\ Southampton General Hospital, Southampton
}

SUMMARY The cervical somatosensory evoked potential after median nerve stimulation was recorded in 14 patients with cervical spondylosis with radiculopathy and, in 10 cases, myelopathy. Four patients had normal potentials, five showed delayed potentials, and in five others no potential could be seen.

The cervical somatosensory evoked potential (CSEP) has been recorded after median nerve stimulation at the wrist in normal subjects (Matthews et al., 1974; Cracco and Cracco, 1976; Jones, 1977; El-Negamy and Sedgwick, 1978) from electrodes placed over the seventh cervical vertebra and a midfrontal scalp electrode $(\mathrm{Fz}$ on the 10-20 system). The potential consists of four negative peaks called N9, N11, N13, and N14 according to their polarity and latency, and is terminated by the N20 potential which is the first cortical event.

The first component (N9) of the CSEP is generated by the afferent volley as it traverses the medial cord of the brachial plexus (Jones, 1977) while the subsequent peak (N11) is of spinal origin from the dorsal horn of the spinal cord (ElNegamy and Sedgwick, 1978). The N13 and N14 peaks originate from more rostral structures, and the nucleus cuneatus and the thalamus are likely generators but there is no clear indication of the precise site.

Abnormalities in the CSEP have been reported in a high proportion of patients with multiple sclerosis and can be a useful aid to diagnosis (Small, 1975; Mastaglia et al., 1976; Small et al., 1978). A prolonged latency was a relatively uncommon finding in multiple sclerosis, reduced amplitudes and abnormal waveforms being seen more often. In this paper we examine the CSEP of a small group of patients with radiculopathy and myelopathy caused by cervical spondylosis.

\footnotetext{
Address for reprint requests: Dr E. M. Sedgwick, Department of Clinical Neurophysiology, Wessex Neurological Centre, Southampton General Hospital, Southampton SO9 4XY.

Accepted 25 August 1978
}

\section{Methods}

\section{RECORDING}

Silver-silver chloride discs were attached to the skin at $\mathrm{Fz}$ position on the scalp and just rostral to the seventh cervical spine $(\mathrm{Cv} 7)$ for recording. In some subjects additional recording sites were used such as Erb's point, to time the arrival of the afferent volley, and C 3 or C4 scalp electrodes (10-20 system) to record the early part of the cortical somatosensory evoked potential. Electrode resistances were low, less than $2 \mathrm{kohm}$, and the subjects were relaxed during recording to limit myogenic potentials. Stimulation at $2 / \mathrm{sec}$ was by a $0.2 \mathrm{~ms}$ rectangular electric pulse delivered to the median nerve at the wrist through an $\mathrm{Ag}-\mathrm{AgCl}$ disc overlying the nerve and an ECG plate electrode on the dorsum of the wrist. A stimulus strength of three times sensory threshold was used.

The signal was amplified by Brookdeal Ortec 9454 amplifiers with a bandwidth of $10 \mathrm{~Hz}-10 \mathrm{kHz}$ and recorded on magnetic tape using an FM system. A trigger pulse marking stimulation time was recorded on a separate channel. The signal was analysed by off-line averaging using a PDP-12 computer with programs ECAN and ECOGB kindly supplied by $\operatorname{Dr} A$. R. D. Thornton. The potentials were plotted by an incremental plotter, and amplitude and latency measurements of the peaks were made by eye and by the computer. Full details of the method have been published (El-Negamy and Sedgwick, 1978).

\section{SUBJECTS}

Normal data were obtained from 31 subjects with no neurological illness. Fourteen patients, four of 
them women, of ages 31-76 years were admitted for investigation of neurological symptoms and, after clinical examination and necessary investigations, a diagnosis of radiculopathy and/or myelopathy secondary to cervical spondylosis was made. All patients gave their informed consent for this procedure which had the approval of the Ethical Committee.

\section{Results}

A normal CSEP is shown in Fig. 1A, trace 1. It is derived from an electrode at $\mathrm{Cv} 7$ and the negative peaks are labelled according to their latency. Peak N9 is generated by the compound action potential in the medial cord of the brachial plexus, and trace A2 shows the potential as it passes Erb's point. Both these events precede N11 which is generated in the dorsal horn of the spinal cord (El-Negamy and Sedgwick, 1978).

Figure $1 \mathrm{~B}$ is from a patient with cervical spondylosis with a mild radiculopathy and myelopathy. The response is reduced in amplitude and delayed in time but the individual components can still be identified. The N9 component and the Erb's point potential are normal.
Fourteen patients with cervical spondylosis were examined. Five showed no clearly identified CSEP except for the N9 potential. The results in the other nine are given in Fig. 2 which also summarises the amplitudes and latencies of the CSEP components in normal subjects.

There is some variation in the latency of the potentials because of different arm lengths. This can be overcome by measuring all latencies from the peak of N9 which is generated in the brachial plexus. The amplitude versus latency of N11, N13, and N14 of normal subjects is shown in the form of three ellipses constructed so that the amplitude/ latency points of $90 \%$ of normal subjects would be expected to fall within them. The individual points for nine patients with cervical spondylosis were plotted, and they fall clearly into two groups. The first group of four shows potentials within the normal range while the second group had low amplitude and delayed potentials like the one shown in Fig. 1. The remaining five patients all had a normal N9 potential but subsequent waves could not be identified. The latencies of the potentials of the second group are shown in the Table. The latency of N9 was normal but there were statistically significant delays between N9 and N11,

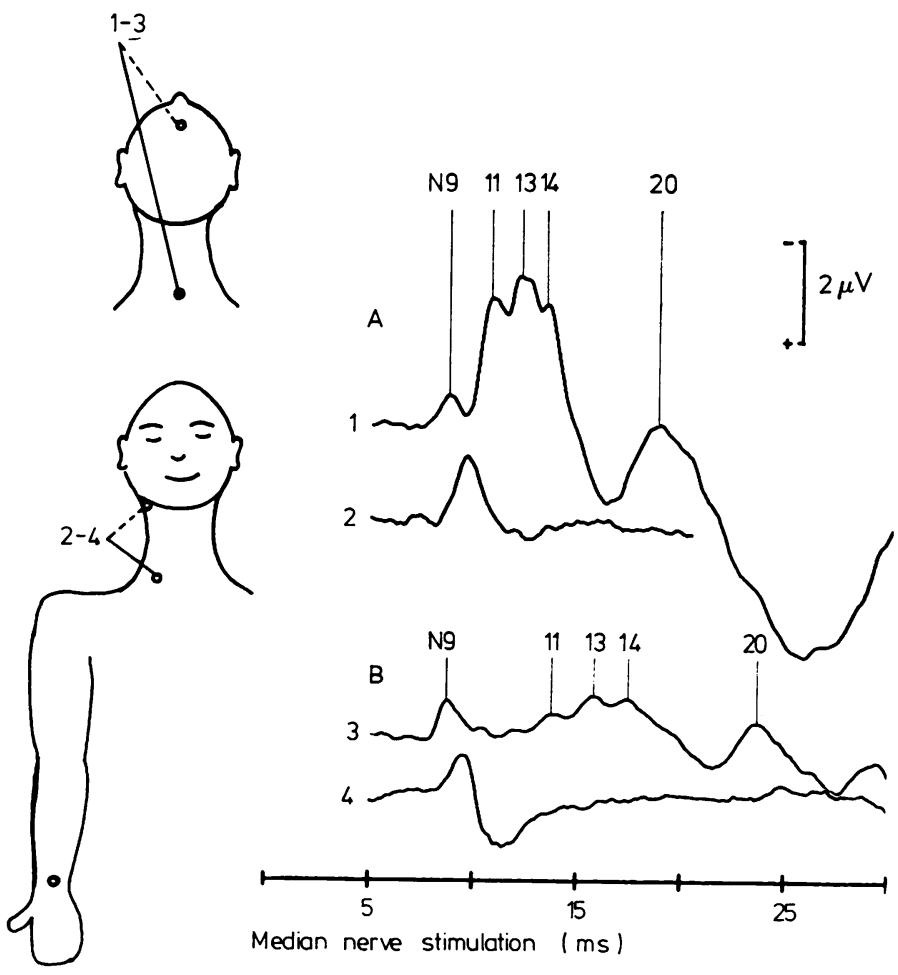

Fig. 1 The cervical somatosensory evoked potential recorded from electrodes on the seventh cervical vertebra and $F z$ and the afferent volley potential from Erb's point of a normal subject are shown in $A$. The different components of the waveform are labelled. In $B$ the recording is from a subject with cervical spondylosis with radiculopathy and myelopathy. N9 and the Erb's point potential have normal amplitude and latency but the later potentials are delayed. A negative potential recorded at the active electrode (Cv7 and Erb's point respectively) gives an upward deflection. Calibration, $2 \mu V$, applies to all four traces. 


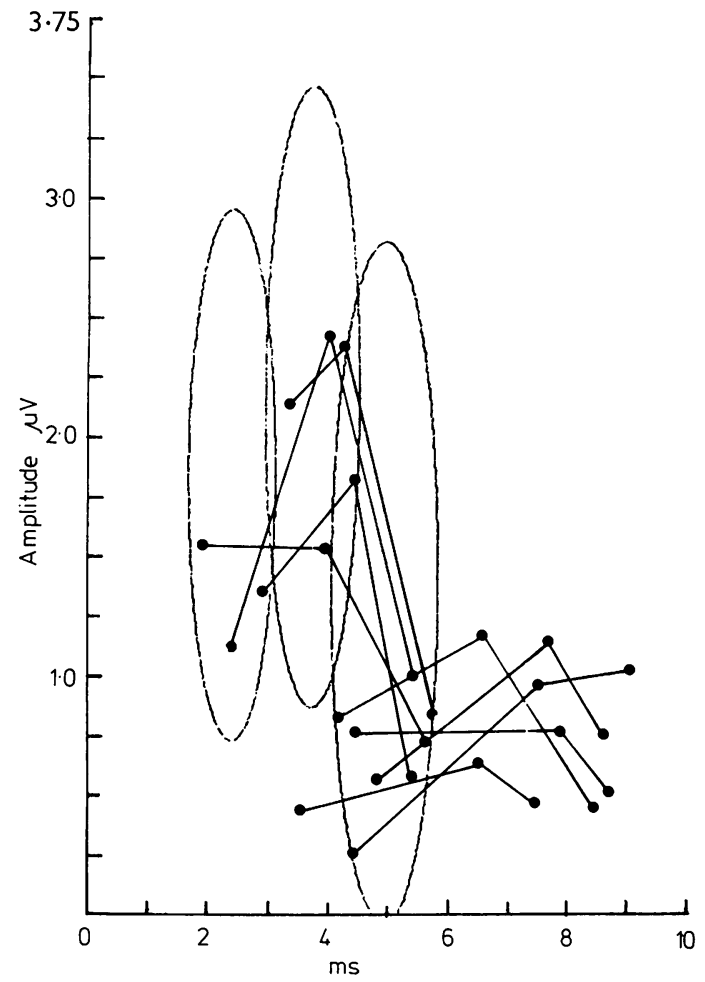

Fig. 2 The amplitudes and latencies of N11,N13, and $N 14$ were measured from the peak of N9 in 31 normal subjects and are expressed as ellipses which mark the $90 \%$ confidence limits. The lines join the amplitudes and latencies of the peaks seen in nine patients with cervical spondylosis. In four cases the peaks fall within the normal range but in five they were delayed. In another five no clear potentials were identified after N9.

$\mathrm{N} 11$ and N13, and N9 and N20. The N13 to N14 interval was normal.

As the brachial plexus potential (N9) was normal in amplitude and latency in this group there must have been normal conduction of the afferent volley of impulses after stimulation up to the medial cord of the brachial plexus. There was an increased delay from this point to the spinal cord as shown by the N9-N11 latency, and a further delay between N11 and N13 indicating a prolonged conduction time to the generator of $\mathrm{N} 13$ which is thought to lie rostral to the cord, possibly the nucleus cuneatus. The prolonged latency at the cerebral cortex as shown by the latency of the N20 wave reflects the delays at the lower levels.

The four patients with normal CSEPs all had symptoms of radiculopathy and evidence on myelography of involvement of the sixth and seventh
Table Mean latencies to the peaks of the negative waves of the CSEP in normal subjects and in five patients with cervical spondylosis who had abnormal CSEP. The statistical significance $(P)$ was calculated by Wilcoxon's rank test. $N S=$ not significant

\begin{tabular}{lccc}
\hline CSEP & $\begin{array}{l}\text { Normal subjects } \\
\text { latency }(\mathrm{ms})\end{array}$ & $\begin{array}{l}\text { Cervical spondylosis } \\
\text { latency }(\mathrm{ms})\end{array}$ & $P$ \\
\hline Stim-N9 & $\mathrm{n}=31$ & $\mathrm{n}=5$ & \\
N9-N11 & $9.04 \pm 0.80$ & $9.24 \pm 0.25$ & $\mathrm{NS}$ \\
N11-N13 & $2.40 \pm 0.45$ & $4.12 \pm 0.49$ & 0.01 \\
N13-N14 & $1.35 \pm 0.44$ & $2.90 \pm 0.38$ & 0.01 \\
N9-N20 & $1.22 \pm 0.34$ & $1.32 \pm 0.47$ & $\mathrm{NS}$ \\
& $10.70 \pm 0.91$ & $12.50 \pm 0.76$ & 0.01 \\
\hline
\end{tabular}

intervertebral foramina. All had objective sensory loss, and three had weakness of the arm muscles. One of this group of patients had evidence of myelopathy with sensory changes and spasticity in the lower limbs. Myelography showed a block at $\mathrm{C} 4 / 5$ intervertebral space.

The patients with delayed potentials all had clinical evidence of both radiculopathy and myelopathy but all were able to walk, although one needed assistance. Radiology demonstrated severe cervical degenerative change, and positive contrast myelography done in four out of five patients showed complete or partial block in the cervical region. Myelography was not attempted in the fifth patient because of his age.

One of the five patients who had no distinguishable CSEP had signs of a severe radiculopathy affecting C6, 7 and 8 roots. There was no evidence of myelopathy, and myelography was not done. The remaining patients had signs of radiculopathy and myelopathy with complete or partial block demonstrated by myelography. All of them could walk without assistance and they were not more severely affected than patients with delayed potentials.

\section{Discussion}

The presence of a normal N9 potential in all patients indicates that a normal afferent volley reached the brachial plexus without delay. The delayed development of the N11 potential, therefore, shows prolonged conduction time between the brachial plexus and the spinal cord.

All the patients with delayed N11 potentials had radiculopathy. The compressed nerve roots would be expected to behave as nerve trunks in entrapment neuropathies, that is by loss of some axons, and thinning and demyelination of others which produces slowed conduction and reduced synchrony of an afferent volley. As the N11 potential is of spinal origin, the involved roots would produce a delayed N11 potential because of slowed 
conduction, and a low amplitude because of loss or conduction block of axons and reduced synchrony of the volley.

There is evidence that $\mathrm{N} 13$ is generated more rostrally, possibly in the cuneate nucleus (Jones, 1977). The timing of N13 with respect to N11 would, therefore, reflect the conduction velocity in the dorsal column fibres. Our finding of an increased delay between $\mathrm{N} 11$ and $\mathrm{N} 13$ suggests that there is additional slowing of conduction in the dorsal column fibres. It is well known that white matter destruction and dorsal column degeneration occur in cervical spondylotic myelopathy (Wilkinson, 1960; Hughes, 1966), and it is not unreasonable to suggest that conduction velocity in the dorsal column fibres is reduced by damage caused by the continual friction between cord and canal wall. All the patients showing this delay had myelopathy.

The N13-N14 interval is normal in all cases. The N14 potential is presumed to have a subcortical origin above the medulla. A further delay here would not be expected. The $\mathrm{N} 20$ potential follows and is delayed in total time because of the conduction delays already indicated in the roots and dorsal columns. This explanation of the abnormalities of the CSEP is consistent with the pathology but it is clearly important to be sure of the generator sites of the CSEP before one can take interpretation in greater depth.

It has been shown that an abnormal CSEP can result from a clinically silent lesion in multiple sclerosis (Small, 1975; Mastaglia et al., 1976; Small et al., 1978). The CSEP may be normal or absent in both multiple sclerosis and cervical spondylosis but in the latter condition a delay between N9 and N11 may be seen, a feature which has not been reported in multiple sclerosis. If our interpretation of the delayed response is correct this feature would be expected in conditions in which radiculopathy or myelopathy or both occur.
We are grateful to $\operatorname{Dr} A$. R. D. Thornton for his help with computer programs, to the consultant staff of the Wessex Neurological Centre for permission to see their patients, and to the patients themselves. Professor K. A. Munday of the Department of Physiology and Biochemistry kindly gave computer time.

\section{References}

Cracco, R. G., and Cracco, J. B. (1976). Somatosensory evoked potentials in man: far field potentials. Electroencephalography and Clinical Neurophysiology, 41, 460-466.

El-Negamy, E., and Sedgwick, E. M. (1978). Properties of a spinal somatosensory evoked potential recorded in man. Journal of Neurology, Neurosurgery, and Psychiatry, 41, 762-768.

Hughes, J. T. (1966). Pathology of the Spinal Cord. Lloyd Luke: London.

Jones, S. J. (1977). Short latency potentials recorded from the neck and scalp following median nerve stimulation in man. Electroencephalography and Clinical Neurophysiology, 43, 853-863.

Mastaglia, F. L., Black, J. L., and Collins, D. W. K. (1976). Visual and spinal evoked potentials in diagnosis of multiple sclerosis. British Medical Journal, 2, 732.

Matthews, W. B., Beauchamp, M., and Small, D. G. (1974). Cervical somatosensory evoked responses in man. Nature, 252, 230-232.

Small, D. G. (1975). Peripherally evoked spinal cord potential in neurological diagnosis. In Scientific Aids in Hospital Diagnosis, pp. 155-163. Edited by J. P. Nicholson. Plenum: New York.

Small, D. G., Matthews, W. B., and Small, M. (1978). The cervical somatosensory potential (SEP) in the diagnosis of multiple sclerosis. Journal of the Neurological Sciences, 35, 211-244.

Wilkinson, M. (1960). The morbid anatomy of cervical spondylosis and myelopathy. Brain, 83, 589-617. 